\title{
MODELAGEM TERMODINÂMICA POR EXTRAÇÃO POR SOLVENTE DE METAIS DIVALENTES EM MEIO SULFATO USANDO D2EHPA
}

Clenilson da Silva Sousa Junior* e Dalton de Sousa Ximenes

Departamento de Química, Instituto Federal de Educação, Ciência e Tecnologia do Rio de Janeiro, Campus Maracanã, Rua Senador Furtado, 121, 20270-021 Rio de Janeiro - RJ, Brasil

Marisa Nascimento e Karina Rodrigues Paiva Ranauro do Nascimento

Coordenação de Processos Metalúrgicos e Ambientais, Centro de Tecnologia Mineral, Av. Pedro Calmon, 900, 21941-908 Rio

de Janeiro - RJ, Brasil

\section{Osvaldo Galvão Caldas da Cunha}

Departamento de Processos Inorgânicos, Escola de Química, Universidade Federal do rio de Janeiro, Av. Horácio Macedo, 2030, Bl. E, 21941-909 Rio de Janeiro - RJ, Brasil

Recebido em 16/2/11; aceito em 5/8/11; publicado na web em 30/9/11

\begin{abstract}
THERMODYNAMIC MODELING FOR SOLVENT EXTRACTION OF METALS DIVALENT IN SULPHATE MEDIA USING D2EHPA. The extraction of divalent metals $\left(\mathrm{Mn}^{2+}, \mathrm{Ni}^{2+}, \mathrm{Co}^{2+}\right.$ and $\left.\mathrm{Cu}^{2+}\right)$ in the system $\mathrm{MSO}_{4}-\mathrm{H}_{2} \mathrm{SO}_{4}-\mathrm{H}_{2} \mathrm{O}-\mathrm{D} 2 \mathrm{EHPA}$ in isoparaffin (17/21) was studied by a thermodynamic model based on chemical equilibria with mass and charge balance equations. The activity coefficients of all solutes in the aqueous phase were calculated by Davies equation. By applying this model, the equilibrium concentrations of solutes were calculated from de concentration of divalent metals and $\mathrm{pH}$. The predicted distribution coefficients for the divalents metals were in good agreement with experimental results.
\end{abstract}

Keywords: modeling; solvent extraction; divalent metals.

\section{INTRODUÇÃO}

A extração por solventes, também conhecida como extração líquido-líquido, é a operação unitária na qual uma fase aquosa, contendo as espécies metálicas a serem separadas é contatada com uma fase orgânica imiscível que extrai as espécies seletivamente. É uma operação unitária hidrometalúrgica que utiliza, muitas vezes, soluções aquosas concentradas, podendo ser empregada na purificação seletiva de licores de lixiviação e efluentes, além da concentração metálica (caso seja necessário, na etapa de re-extração dos metais da fase orgânica). A distribuição dos íons metálicos entre as fases aquosa e orgânica depende de uma série de variáveis, tais como, composição da solução, $\mathrm{pH}$, concentração do extratante, relação do volume de fases aquosa/orgânica (A/O), tipo de diluente e tempo de contato entre as fases e agitação. A grande vantagem da extração por solventes é a redução da geração global de resíduos de processo, permitindo uma elevada recuperação do metal de interesse e o reciclo do solvente usado no processo..$^{1-4}$ Esse processo associado a ferramentas de modelagem e simulação possibilita esclarecer os processos de separação e purificação de elementos ou compostos minerais com elevado grau de pureza. ${ }^{5}$ A técnica de extração por solvente possui aplicações na indústria nuclear, na mineração, na metalurgia, no tratamento de efluentes industriais tanto para desenvolvimento de novos projetos, como para dinamizar ou incrementar instalações já existentes. ${ }^{6-10}$

A modelagem matemática consiste em partir de um fato real ou semirreal e criar, por meio da coleta, análise e organização dos dados coletados, uma expressão em linguagem matemática que possa servir de parâmetro para descrição e compreensão da realidade. Na visão de Bienbengut e Hein, ${ }^{11}$ a interação que permite transformar uma situação real em um "modelo matemático" pertinente deve seguir três etapas básicas: a interação, onde se tem reconhecimento do problema e a familiarização com o fenômeno a ser modelado; a matematização,

*e-mail: clenilsonjunior@gmail.com momento em que é realizada a formulação do problema e, o modelo propriamente dito, que interpreta a solução e valida a expressão matemática desenvolvida.

Numerosos modelos matemáticos têm sido desenvolvidos para o cálculo do coeficiente de atividade de íons, principalmente para soluções hidrometalúrgicas, onde a correção da concentração utilizando o coeficiente de atividade é um fato primordial. O modelo mais tradicional é o de Debye-Hückel, porém não é aplicável quando se trata de soluções concentradas, sendo válido para valores de força iônica de até $100 \mathrm{mmol} / \mathrm{L}$, apresentando grande desvio para valores experimentais acima disso. ${ }^{12,13}$ Mais recentemente, alguns autores demonstraram com bastante clareza os efeitos da atividade química em eletrólitos concentrados podendo-se destacar, nesse sentido, os trabalhos de Samson ${ }^{6}$ e Pitzer, ${ }^{7}$ que descrevem modelos semiempíricos que são aplicáveis para soluções com forças iônicas superiores a $1000 \mathrm{mmol} / \mathrm{L}$.

Este trabalho teve como objetivo desenvolver uma metodologia para o modelamento da extração de metais divalentes em meio sulfúrico, utilizando como extratante o ácido Di-2-etil-hexil fosfórico, conhecido como D2EHPA, dissolvido em isoparafina (17/21), com base em equações de equilíbrio, balanço de massa e carga. Os coeficientes de atividade de todos os solutos na fase aquosa foram considerados no modelo. As concentrações das espécies no equilíbrio foram calculadas pelo método de Newton-Raphson, de forma a se determinar as condições reacionais no equilíbrio. ${ }^{14}$

Além do D2EHPA, destacam-se outros extratantes como o Cyanex 302 (ácido bis (2,4,4-trimetil pentil) monotiofosfínico) e o Cyanex 272 (ácido bis (2,4,4-trimetil pentil fosfínico), ambos da Cytec (USA), que apresentam basicamente o mesmo mecanismo reacional de troca catiônica. ${ }^{15}$ No caso do extratante D2EHPA, a reação entre os íons metálicos com o extratante, dissolvido em diluentes alifáticos, é comumente descrita na literatura internacional segundo a Equação 1: ${ }^{16}$

$$
M_{(a q)}^{2+}+n(H A)_{2(o r)} \rightarrow M A_{2}(H A)_{2(n-1)(o r)}+2 H_{(a q)}^{+}
$$


onde (HA) $)_{2}$ representa a forma das moléculas de D2EHPA e $\mathrm{M}^{2+}$ representa qualquer metal divalente. $\mathrm{O}$ valor de $n$ dependerá dos fatores de processo como tipo de meio, forma do diluente, concentração, etc.

Supondo que a molécula de D2EHPA exista como um dímero $\left(\mathrm{H}_{2} \mathrm{~A}_{2}\right)$ em solventes alifáticos, como a isoparafina, a extração da reação de M(II) com D2EHPA pode ser escrita de acordo com a Equação $2: 17,18$

$M_{(a q)}^{2+}+(1+x) H_{2} A_{2(a q)} \rightarrow M H_{2 x} A_{2+2 x(o r)}+2 H_{(a q)}^{+}$

O coeficiente de distribuição (D) do metal pode ser calculado através da razão entre a concentração total $(\mathrm{mmol} / \mathrm{L})$ do metal na fase orgânica sobre a concentração total do metal na fase aquosa, conforme a Equação 3:19

$D=\frac{\left[M H_{2 x} A_{2+2 x}\right]_{(o r, t)}}{\left[M^{2+}\right]_{(a q, t)}}$

A constante de equilíbrio $\left(\mathrm{K}_{\mathrm{eq}}\right)$ para a reação de extração pode ser representada por meio da Equação 4:

$K e q=\frac{\left[M H_{2 x} A_{2+2 x}\right] \cdot\left[H^{+}\right]^{2}}{\left[M^{2+}\right] \cdot\left[H_{2} A_{2}\right]^{(1+x)}} x \frac{\gamma_{M H_{2 x} A_{2+2 x}} \cdot \gamma_{H^{+}}^{2}}{\gamma_{M^{2+}} \cdot \gamma_{H_{2} A_{2}}^{(1+x)}}$

Aplicando o logaritmo em ambos os lados da Equação 4, após incluir a Equação 3 na Equação 4, obtêm-se a Equação 5:

$\log D-2 p H=(1+x) \log \left[H_{2} A_{2}\right]+\log K_{e q}-\log \frac{\gamma_{M H_{2 x} A_{2+2 x}} \cdot \gamma_{H^{+}}^{2}}{\gamma_{M^{2+}} \cdot \gamma_{H_{2} A_{2}}^{(1+x)}}$

A concentração de $\mathrm{H}_{2} \mathrm{~A}_{2}$ na Equação 5 no equilíbrio é dada pela Equação 6:

$\left[H_{2} A_{2}\right]=\left[H_{2} A_{2}\right]_{\text {inicial }}-2(1+x)\left[M H_{2 x} A_{2+2 x}\right]$

Por fim, excluindo-se o último termo da Equação 5 e adicionandose a Equação 6 na Equação 5, obtém-se a Equação 7:

$\log D-2 p H=(1+x) \log \left\{\left[H_{2} A_{2}\right]_{\text {inicial }}-2(1+x)\left[M H_{2 x} A_{2+2 x}\right]\right\}+\log K_{\text {eq }}$

\section{PARTE EXPERIMENTAL}

As soluções de sulfato dos quatro metais divalentes foram preparadas pela dissolução de $\mathrm{MnSO}_{4} \cdot 4 \mathrm{H}_{2} \mathrm{O}, \mathrm{NiSO}_{4} \cdot 6 \mathrm{H}_{2} \mathrm{O}, \mathrm{CoSO}_{4} \cdot 7 \mathrm{H}_{2} \mathrm{O}$ e $\mathrm{CuSO}_{4} \cdot 5 \mathrm{H}_{2} \mathrm{O}$ (Vetec) em água deionizada. $\mathrm{O}$ pH da solução aquosa foi condicionado inicialmente com a adição de solução de ácido sulfúrico $\left(\mathrm{H}_{2} \mathrm{SO}_{4}\right)$. A solução de D2EHPA (Rhodia) foi preparada pela dissolução em isoparafina 17/21 (Unipar) sem nenhuma purificação inicial.

Alíquotas de iguais volumes $(25,00 \mathrm{~mL})$ das soluções aquosas $\left(\mathrm{V}_{\mathrm{aq}}\right)$ e orgânicas $\left(\mathrm{V}_{\mathrm{or}}\right)$ foram contatadas em um béquer de vidro de $100 \mathrm{~mL}$ e agitadas magneticamente, com auxílio de uma placa de agitação da marca Ika, em velocidade adequada por 5 min à temperatura de 298,15 K. Depois de decorrido esse tempo de contato, as fases aquosas e orgânicas foram decantadas por 40 min e separadas em funil de decantação. ${ }^{20}$

As concentrações dos metais divalentes da fase aquosa $\left(\left[\mathrm{M}^{2+}\right]_{\mathrm{aq}, \mathrm{f}}\right)$ foram determinadas por espectroscopia de absorção atômica (Spectra model 50B) com grau de incerteza de $10^{-4}$ e as concentrações da fase orgânica $\left(\left[\mathrm{MH}_{2 x} \mathrm{~A}_{2+2 x}\right]\right)$ foram determinadas usando-se balanço de massa para cada espécie formada. $\mathrm{O} \mathrm{pH}$ foi medido usando um pHmetro (Digimed).

\section{RESULTADOS E DISCUSSÃO}

\section{Constante de equilíbrio a determinação do complexo metálico}

As Tabelas 1 a 4 apresentam os resultados experimentais dos testes de extração. Duas incógnitas, $\mathrm{x}$ e $\mathrm{K}_{\text {eq }}$, podem ser determinadas utilizando a Equação 7. O método de estimativa não linear QuasiNewton, realizado utilizando o software Statistica ${ }^{\circledR}$, foi aplicado aos resultados obtidos da absorção atômica e foram encontrados valores para x e $\mathrm{K}_{\mathrm{eq}}$ como pode ser observado na Tabela 5, obtendose coeficientes de determinação $\left(\mathrm{R}^{2}\right)$ com valores adequados para manganês, níquel, cobalto e cobre. Com os valores de x determinados, as espécies extraídas para a fase orgânica podem ser representadas como $\mathrm{MnH}_{0,2} \mathrm{~A}_{2,2}, \mathrm{NiH}_{0,25} \mathrm{~A}_{2,25}, \mathrm{CoH}_{0,25} \mathrm{~A}_{2,25}$ e $\mathrm{CuH}_{2,4} \mathrm{~A}_{4,4}$.

De acordo com Ritcey e Ashbrook, ${ }^{22}$ algumas considerações devem ser levadas em conta na extração por solvente, quando se utiliza um extratante organofosforado. Para metais com a mesma carga, a extração é inversamente proporcional ao raio iônio (Tabela 5), isto é, quanto menor o íon, mais efetiva será a extração. Este fato foi observado na extração dos metais divalentes com o D2EHPA, pois os raios iônicos dos metais divalentes decrescem na seguinte ordem: $\mathrm{Mn}^{2+}>\mathrm{Ni}^{2+}>\mathrm{Co}^{2+}>\mathrm{Cu}^{2+}$. Sendo assim, o $\mathrm{Cu}^{2+}$ é o íon extraído com maior facilidade, seguindo pelo $\mathrm{Co}^{2+} \mathrm{e} \mathrm{Ni}^{2+} \mathrm{e}$, por último, o íon $\mathrm{Mn}^{2+}$. Esta teoria é confirmada pelas espécies orgânicas extraídas (Equações 8 a 11) e pelos valores de $K_{e q}$ determinados.

$$
\begin{aligned}
& \mathrm{Mn}_{(a q)}^{2+}+1.1 \mathrm{H}_{2} \mathrm{~A}_{2(o r)} \rightarrow \mathrm{MnH}_{02} A_{2.2(\text { org })}+2 \mathrm{H}_{(a q)}^{+} \\
& \mathrm{Ni}_{(a q)}^{2+}+1.125 \mathrm{H}_{2} A_{2(o r)} \rightarrow \mathrm{NiH}_{0.25} A_{2.25(\text { org })}+2 H_{(a q)}^{+} \\
& \mathrm{Co}_{(a q)}^{2+}+1.125 \mathrm{H}_{2} A_{2(o r)} \rightarrow \mathrm{CoH}_{025} A_{225(\text { org })}+2 H_{(a q)}^{+} \\
& \mathrm{Cu}_{(a q)}^{2+}+2.2 \mathrm{H}_{2} \mathrm{~A}_{2(\mathrm{or})} \rightarrow \mathrm{CuH}_{2.4} A_{4.4(\text { org })}+2 \mathrm{H}_{(a q)}^{+}
\end{aligned}
$$

\section{Modelo termodinâmico}

O número de solutos na fase aquosa antes da extração é de dez $\left(\mathrm{M}^{2+}, \mathrm{MSO}_{4}, \mathrm{MHSO}_{4}^{+}, \mathrm{M}(\mathrm{OH})^{+}, \mathrm{M}(\mathrm{OH})_{2}, \mathrm{H}^{+}, \mathrm{OH}^{-}, \mathrm{SO}_{4}{ }^{2-}, \mathrm{HSO}_{4}^{-} \mathrm{e}\right.$ $\mathrm{H}_{2} \mathrm{O}$ ) e as constantes utilizadas para a construção do modelo termodinâmico podem ser verificadas na Tabela 6 .

Dez equações independentes são necessárias para resolver as dez variáveis desconhecidas. Primeiramente, a atividade da água é considerada um como uma aproximação, igual a 1. Em segundo lugar, a atividade do hidrogênio é obtida a partir do valor de $\mathrm{pH}$ inicial. As atividades dos oito solutos restantes são calculadas pela solução das equações de seis equilíbrios químicos, um balanço de massa do metal divalente e um balanço de carga simultaneamente (Tabela 6).

Os valores dos coeficientes de atividade e a força iônica foram determinados a partir da Equação de Davies modificada (Equação 12) para cada íon.

$\ln \gamma=\frac{-A_{i}^{2} \sqrt{I}}{1+3 \times 10^{-10} B \sqrt{I}}+\frac{\left(\left(-4,17 \times 10^{-15}\right) I+0,2\right) A z_{i}^{2} I}{\sqrt{1000}}$

Os parâmetros A e B da Equação 12 são apresentados nas Equações 13 e 14:

$$
\begin{gathered}
A=\frac{\sqrt{2 F^{2} e_{0}}}{8 \pi(R T)^{3 / 2}} \\
B=\frac{\sqrt{2 F^{2}}}{\varepsilon R T}
\end{gathered}
$$

onde $\mathrm{e}_{\mathrm{o}}$ é a carga do elétron $\left(\mathrm{e}_{\mathrm{o}}=1,602 \times 10^{-19} \mathrm{C}\right) ; \varepsilon=\varepsilon_{\mathrm{r}} \varepsilon_{0}$ é a 
Tabela 1. Dados experimentais e calculados para extração de manganês

\begin{tabular}{|c|c|c|c|c|c|c|c|c|}
\hline Teste & $\begin{array}{l}{\left[\mathrm{Mn}^{2+}\right]_{\mathrm{i}}} \\
(\mathrm{mol} / \mathrm{L})\end{array}$ & $\begin{array}{c}{\left[\mathrm{Mn}^{2+}\right]_{\text {aq. f }}} \\
(\mathrm{mol} / \mathrm{L})\end{array}$ & $\begin{array}{c}{[\mathrm{HA}]^{\mathrm{i}}} \\
(\mathrm{mol} / \mathrm{L}) \\
\end{array}$ & $\mathrm{pH}_{\mathrm{i}}$ & $\log D_{\text {exp. }}$ & $\log D_{\text {calc. }}$ & $\mathrm{pH}_{\text {exp. eq. }}$ & $\mathrm{pH}_{\text {calc. eq. }}$ \\
\hline 1 & 0,00805 & 0,00685 & 0,05 & 2,30 & $-0,7570$ & $-0,7516$ & 2,13 & 2,09 \\
\hline 2 & 0,00909 & 0,00565 & 0,05 & 3,24 & $-0,2161$ & $-0,2162$ & 2,40 & 2,43 \\
\hline 3 & 0,00867 & 0,00545 & 0,05 & 4,02 & $-0,2269$ & $-0,2394$ & 2,35 & 2,36 \\
\hline 4 & 0,00805 & 0,00591 & 0,10 & 2,30 & $-0,4425$ & $-0,4665$ & 2,05 & 2,07 \\
\hline 5 & 0,00909 & 0,00508 & 0,10 & 3,24 & $-0,1029$ & $-0,1040$ & 2,27 & 2,26 \\
\hline 6 & 0,00867 & 0,00478 & 0,10 & 4,02 & $-0,0883$ & $-0,0920$ & 2,24 & 2,27 \\
\hline 7 & 0,01300 & 0,01170 & 0,05 & 3,05 & $-0,9747$ & $-0,9406$ & 2,22 & 2,22 \\
\hline 8 & 0,01290 & 0,01140 & 0,05 & 4,07 & $-0,8928$ & $-0,9018$ & 2,30 & 2,32 \\
\hline 9 & 0,01310 & 0,01230 & 0,10 & 2,00 & $-1,2291$ & $-1,2429$ & 1,86 & 1,85 \\
\hline 10 & 0,01300 & 0,01070 & 0,10 & 3,05 & $-0,6696$ & $-0,6717$ & 2,07 & 2,04 \\
\hline 11 & 0,01290 & 0,01020 & 0,10 & 4,07 & $-0,5751$ & $-0,5895$ & 2,13 & 2,15 \\
\hline 12 & 0,01310 & 0,01110 & 0,20 & 2,00 & $-0,7496$ & $-0,7560$ & 1,80 & 1,93 \\
\hline 13 & 0,01300 & 0,00891 & 0,20 & 3,05 & $-0,3420$ & $-0,3219$ & 1,97 & 2,00 \\
\hline 14 & 0,01290 & 0,00912 & 0,20 & 4,07 & $-0,3872$ & $-0,3708$ & 1,98 & 2,03 \\
\hline 15 & 0,02570 & 0,02390 & 0,10 & 2,04 & $-1,1051$ & $-1,1694$ & 1,88 & 1,83 \\
\hline 16 & 0,02570 & 0,02240 & 0,10 & 3,01 & $-0,8268$ & $-0,8268$ & 2,07 & 2,07 \\
\hline 17 & 0,02490 & 0,02200 & 0,10 & 4,04 & $-0,8794$ & $-0,8268$ & 2,06 & 2,07 \\
\hline 18 & 0,02570 & 0,02220 & 0,20 & 2,04 & $-0,7986$ & $-0,8077$ & 1,80 & 1,84 \\
\hline 19 & 0,02570 & 0,02010 & 0,20 & 3,01 & $-0,5544$ & $-0,5699$ & 1,91 & 1,91 \\
\hline 20 & 0,02490 & 0,02050 & 0,20 & 4,04 & $-0,6716$ & $-0,6732$ & 1,93 & 1,92 \\
\hline
\end{tabular}

Tabela 2. Dados experimentais e calculados para extração de níquel

\begin{tabular}{|c|c|c|c|c|c|c|c|c|}
\hline Teste & $\begin{array}{c}{\left[\mathrm{Ni}^{2+}\right]_{\mathrm{i}}} \\
(\mathrm{mol} / \mathrm{L})\end{array}$ & $\begin{array}{c}{\left[\mathrm{Ni}^{2+}\right]_{\mathrm{f}}} \\
(\mathrm{mol} / \mathrm{L})\end{array}$ & $\begin{array}{c}{[\mathrm{HA}]_{\mathrm{i}}} \\
(\mathrm{mol} / \mathrm{L})\end{array}$ & $\mathrm{pH}_{\mathrm{i}}$ & $\log D_{\text {exp. }}$ & $\log \mathrm{D}_{\text {calc. }}$ & $\mathrm{pH}_{\text {exp. eq. }}$ & $\mathrm{pH}_{\text {calc. eq. }}$ \\
\hline 1 & 0,00789 & 0,00708 & 0,05 & 3,84 & $-0,939092$ & $-0,869800$ & 2,89 & 2,83 \\
\hline 2 & 0,00811 & 0,00749 & 0,05 & 4,73 & $-1,084622$ & $-1,018969$ & 2,94 & 2,91 \\
\hline 3 & 0,00800 & 0,00728 & 0,10 & 3,13 & $-1,004009$ & $-1,013074$ & 2,55 & 2,52 \\
\hline 4 & 0,00789 & 0,00728 & 0,10 & 3,84 & $-1,075558$ & $-1,058153$ & 2,71 & 2,72 \\
\hline 5 & 0,00811 & 0,00705 & 0,10 & 5,00 & $-0,823125$ & $-0,771141$ & 2,72 & 2,74 \\
\hline 6 & 0,01360 & 0,01262 & 0,05 & 4,11 & $-1,106396$ & $-1,127802$ & 2,92 & 2,97 \\
\hline 7 & 0,01402 & 0,01293 & 0,05 & 4,89 & $-1,074439$ & $-1,073110$ & 2,89 & 2,90 \\
\hline 8 & 0,01426 & 0,01288 & 0,10 & 3,14 & $-0,970241$ & $-0,984887$ & 2,61 & 2,67 \\
\hline 9 & 0,01419 & 0,01280 & 0,10 & 4,11 & $-0,965247$ & $-0,967904$ & 2,65 & 2,67 \\
\hline 10 & 0,01341 & 0,01262 & 0,10 & 4,75 & $-1,201870$ & $-1,139701$ & 2,67 & 2,63 \\
\hline 11 & 0,01426 & 0,01260 & 0,20 & 3,12 & $-0,879554$ & $-0,837303$ & 2,48 & 2,44 \\
\hline 12 & 0,02627 & 0,02204 & 0,10 & 3,13 & $-0,716165$ & $-0,700344$ & 2,60 & 2,64 \\
\hline 13 & 0,02663 & 0,02125 & 0,10 & 3,98 & $-0,596791$ & $-0,595951$ & 2,69 & 2,74 \\
\hline 14 & 0,02685 & 0,02088 & 0,10 & 4,88 & $-0,543936$ & $-0,560017$ & 2,67 & 2,67 \\
\hline 15 & 0,02627 & 0,02118 & 0,20 & 3,14 & $-0,619090$ & $-0,586984$ & 2,52 & 2,49 \\
\hline 16 & 0,02663 & 0,02127 & 0,20 & 3,96 & $-0,598636$ & $-0,558392$ & 2,25 & 2,20 \\
\hline 17 & 0,02685 & 0,02131 & 0,20 & 5,02 & $-0,585128$ & $-0,577231$ & 2,50 & 2,52 \\
\hline
\end{tabular}

permissividade do meio, dada pela constante dielétrica da água $\left(\varepsilon_{\mathrm{r}}\right)$ que varia com a temperatura e pela permissividade do vácuo $\left(\varepsilon_{\mathrm{o}}=8,854 \times 10^{-12} \mathrm{C}^{2} / \mathrm{J} . \mathrm{m}\right) ; \mathrm{F}$ é a constante de Faraday $(9,64846 \mathrm{x}$ $\left.10^{4} \mathrm{C} / \mathrm{mol}\right)$; R é a constante universal dos gases $(8,3144 \mathrm{~J} / \mathrm{mol} \mathrm{K}) \mathrm{e}$ $\mathrm{T}$ é a temperatura em Kelvin.

Então, um total de oito variáveis e 8 equações forma o sistema que tem sido resolvido pelo método de Newton-Raphson para sistemas de equações lineares. Esse procedimento foi aplicado para determinar a distribuição das espécies na solução, no equilíbrio, antes da extração.

Depois de definir todos os coeficientes de atividade iguais a 1 , as 8 equações são resolvidas e as oito variáveis determinadas. A força iônica é calculada e os coeficientes de atividade também são calculados pela Equação de Davies modificada. Todas as etapas são repetidas até que o critério de convergência (coeficiente calculado igual ao coeficiente estimado) seja satisfeito.
As Figuras 1 a 4 ilustram a distribuição de solutos das espécies que contêm manganês, níquel, cobalto e cobre, em valores de $\mathrm{pH}$ entre 0 e 5, quando a concentração inicial do metal divalente $\left(\left[\mathrm{M}^{2+}\right]_{\mathrm{i}}\right)$ é mantida em $8,05 \mathrm{mmol} / \mathrm{L}$. Na especiação dos solutos de manganês, níquel e cobalto a espécie dominante em $\mathrm{pH}$ baixo é $\mathrm{MHSO}_{4}{ }^{+}\left(\mathrm{MnHSO}_{4}{ }^{+}, \mathrm{NiHSO}_{4}{ }^{+}\right.$e $\left.\mathrm{CoHSO}_{4}{ }^{+}\right)$e da fração livre do metal divalente aumenta com o incremento do $\mathrm{pH}$. Na especiação dos solutos de cobre a espécie dominante em $\mathrm{pH}$ baixo é $\mathrm{CuHSO}_{4}{ }^{+}$ e, após um aumento de $\mathrm{pH}$, as espécies $\mathrm{Cu}^{2+}$ e $\mathrm{CuSO}_{4}$ mantêm faixas de concentração bem próximas. Nos demais casos, com o aumento do valor do $\mathrm{pH}$ ocorre um incremento do percentual das espécies metálicas divalentes, devido à formação dos demais solutos na solução aquosa. Os valores de forças iônicas e coeficientes de atividade para a solução dos quatro metais são apresentados nas Tabelas 7 a 10 
Tabela 3. Dados experimentais e calculados para extração de cobalto

\begin{tabular}{|c|c|c|c|c|c|c|c|c|}
\hline Teste & $\begin{array}{r}{\left[\mathrm{Co}^{2+}\right]_{\mathrm{i}}} \\
(\mathrm{mol} / \mathrm{L}) \\
\end{array}$ & $\begin{array}{c}{\left[\mathrm{Co}^{2+}\right]_{\mathrm{f}}} \\
(\mathrm{mol} / \mathrm{L}) \\
\end{array}$ & $\begin{array}{c}{[\mathrm{HA}]_{\mathrm{i}}} \\
(\mathrm{mol} / \mathrm{L})\end{array}$ & $\mathrm{pH}_{\mathrm{i}}$ & $\log D_{\text {exp. }}$ & $\log D_{\text {calc. }}$ & $\mathrm{pH}_{\text {exp. eq. }}$ & $\mathrm{pH}_{\text {calc. eq. }}$ \\
\hline 1 & 0,00584 & 0,00565 & 0,05 & 3,40 & $-1,469822$ & $-1,512504$ & 2,48 & 2,50 \\
\hline 2 & 0,00622 & 0,00603 & 0,05 & 4,38 & $-1,498311$ & $-1,500671$ & 2,63 & 2,61 \\
\hline 3 & 0,00584 & 0,00546 & 0,10 & 3,40 & $-1,153815$ & $-1,184371$ & 2,49 & 2,46 \\
\hline 4 & 0,00622 & 0,00565 & 0,10 & 4,38 & $-0,992701$ & $-0,982003$ & 2,52 & 2,56 \\
\hline 5 & 0,01044 & 0,01025 & 0,05 & 2,56 & $-1,728354$ & $-1,788516$ & 2,39 & 2,38 \\
\hline 6 & 0,01044 & 0,00986 & 0,10 & 2,56 & $-1,234686$ & $-1,306960$ & 2,33 & 2,37 \\
\hline 7 & 0,01044 & 0,00967 & 0,20 & 2,56 & $-1,101231$ & $-1,109311$ & 2,22 & 2,24 \\
\hline 8 & 0,01005 & 0,00948 & 0,20 & 3,56 & $-1,217484$ & $-1,218556$ & 2,31 & 2,33 \\
\hline 9 & 0,00986 & 0,00929 & 0,20 & 4,62 & $-1,208620$ & $-1,184979$ & 2,33 & 2,42 \\
\hline 10 & 0,02011 & 0,01973 & 0,10 & 2,62 & $-1,711807$ & $-1,721759$ & 2,15 & 2,19 \\
\hline 11 & 0,02049 & 0,01973 & 0,10 & 4,27 & $-1,410777$ & $-1,501209$ & 2,45 & 2,42 \\
\hline 12 & 0,02088 & 0,02011 & 0,20 & 3,53 & $-1,419129$ & $-1,434761$ & 2,25 & 2,27 \\
\hline 13 & 0,00533 & 0,00491 & 0,05 & 3,65 & $-1,075547$ & $-1,138917$ & 2,87 & 2,82 \\
\hline 14 & 0,00574 & 0,00491 & 0,05 & 4,40 & $-0,774517$ & $-0,832014$ & 2,91 & 2,89 \\
\hline 15 & 0,00533 & 0,00450 & 0,10 & 2,78 & $-0,736397$ & $-0,713489$ & 2,61 & 2,63 \\
\hline 16 & 0,00574 & 0,00491 & 0,10 & 4,40 & $-0,774517$ & $-0,751948$ & 2,75 & 2,71 \\
\hline 17 & 0,01111 & 0,00904 & 0,05 & 4,64 & $-0,641474$ & $-0,637323$ & 2,93 & 2,93 \\
\hline 18 & 0,01069 & 0,00987 & 0,10 & 2,86 & $-1,077368$ & $-1,120038$ & 2,47 & 2,46 \\
\hline 19 & 0,01111 & 0,00945 & 0,10 & 4,64 & $-0,757775$ & $-0,724602$ & 2,73 & 2,73 \\
\hline 20 & 0,01069 & 0,00863 & 0,20 & 2,86 & $-0,621176$ & $-0,666925$ & 2,40 & 2,48 \\
\hline 21 & 0,01028 & 0,00863 & 0,20 & 3,77 & $-0,718086$ & $-0,709987$ & 2,57 & 2,59 \\
\hline 22 & 0,01111 & 0,00780 & 0,20 & 4,64 & $-0,373372$ & $-0,358863$ & 2,50 & 2,57 \\
\hline 23 & 0,02308 & 0,01854 & 0,20 & 4,94 & $-0,610854$ & $-0,637097$ & 2,37 & 2,39 \\
\hline
\end{tabular}

Tabela 4. Dados experimentais e calculados para extração de cobre

\begin{tabular}{|c|c|c|c|c|c|c|c|c|}
\hline Teste & $\begin{array}{c}{\left[\mathrm{Cu}^{2+}\right]_{\mathrm{i}}} \\
(\mathrm{mol} / \mathrm{L})\end{array}$ & $\begin{array}{c}{\left[\mathrm{Cu}^{2+}\right]_{\mathrm{f}}} \\
(\mathrm{mol} / \mathrm{L})\end{array}$ & $\begin{array}{c}{[\mathrm{HA}]_{\mathrm{i}}} \\
(\mathrm{mol} / \mathrm{L})\end{array}$ & $\mathrm{pH}_{\mathrm{i}}$ & $\mathrm{pH}_{\mathrm{i}}$ & $\log D_{\text {exp. }}$ & $\log D_{\text {calc. }}$ & $\mathrm{pH}_{\text {exp. eq }}$ \\
\hline 1 & 0,0070 & 0,0068 & 0,05 & 2,02 & $-1,549363$ & $-1,636645$ & 1,48 & 1,48 \\
\hline 2 & 0,0070 & 0,0063 & 0,05 & 2,90 & $-0,973515$ & $-0,972850$ & 1,61 & 1,62 \\
\hline 3 & 0,0071 & 0,0064 & 0,05 & 4,05 & $-0,922068$ & $-0,853652$ & 1,63 & 1,69 \\
\hline 4 & 0,0070 & 0,0063 & 0,10 & 2,02 & $-0,973515$ & $-0,945519$ & 1,33 & 1,31 \\
\hline 5 & 0,0070 & 0,0059 & 0,10 & 2,90 & $-0,750008$ & $-0,709388$ & 1,41 & 1,48 \\
\hline 6 & 0,0071 & 0,0057 & 0,10 & 4,05 & $-0,583153$ & $-0,596500$ & 1,30 & 1,37 \\
\hline 7 & 0,0145 & 0,0137 & 0,05 & 2,04 & $-1,254419$ & $-1,290617$ & 1,57 & 1,53 \\
\hline 8 & 0,0143 & 0,0136 & 0,05 & 3,10 & $-1,309379$ & $-1,310262$ & 1,55 & 1,57 \\
\hline 9 & 0,0145 & 0,0136 & 0,05 & 4,14 & $-1,200234$ & $-1,221827$ & 1,52 & 1,55 \\
\hline 10 & 0,0145 & 0,0132 & 0,10 & 2,04 & $-1,028187$ & $-1,073587$ & 1,43 & 1,53 \\
\hline 11 & 0,0143 & 0,0127 & 0,10 & 3,10 & $-0,892479$ & $-0,840568$ & 1,35 & 1,36 \\
\hline 12 & 0,0145 & 0,0128 & 0,10 & 4,14 & $-0,870915$ & $-0,874168$ & 1,36 & 1,40 \\
\hline 13 & 0,0145 & 0,0120 & 0,20 & 4,14 & $-0,684422$ & $-0,681997$ & 1,16 & 1,14 \\
\hline 14 & 0,0288 & 0,0267 & 0,10 & 2,21 & $-1,103858$ & $-1,139253$ & 1,25 & 1,29 \\
\hline 15 & 0,0288 & 0,0259 & 0,10 & 3,21 & $-0,956544$ & $-0,954365$ & 1,28 & 1,26 \\
\hline 16 & 0,0290 & 0,0261 & 0,10 & 3,72 & $-0,959732$ & $-0,994663$ & 1,43 & 1,42 \\
\hline 17 & 0,0288 & 0,0252 & 0,20 & 2,21 & $-0,840889$ & $-0,840551$ & 1,05 & 1,01 \\
\hline 18 & 0,0288 & 0,0253 & 0,20 & 3,21 & $-0,867655$ & $-0,874168$ & 1,06 & 1,04 \\
\hline 19 & 0,0290 & 0,0248 & 0,20 & 3,72 & $-0,770575$ & $-0,782086$ & 1,13 & 1,17 \\
\hline
\end{tabular}

Tabela 5. Valores determinados pelo método Quasi-Newton de estimativa não linear e raio iônico dos metais divalentes

\begin{tabular}{lcccc}
\hline & $\mathrm{Mn}^{2+}$ & $\mathrm{Ni}^{2+}$ & $\mathrm{Co}^{2+}$ & $\mathrm{Cu}^{2+}$ \\
\hline Raio iônico $\left(10^{-10} \mathrm{~m}\right)^{21}$ & 0,80 & 0,78 & 0,78 & 0,69 \\
Coeficiente estequiométrico $(\mathrm{x})$ & 0,1016 & 0,1233 & 0,1234 & 1,214 \\
Constante de equilíbrio $\left(\mathrm{K}_{\text {eq }}\right)$ & $5,18 \times 10^{-4}$ & $2,40 \times 10^{-5}$ & $2,10 \times 10^{-5}$ & $2,52 \times 10^{-1}$ \\
Coeficiente de determinação $\left(\mathrm{R}^{2}\right)$ & 0,8346 & 0,7377 & 0,8460 & 0,9439 \\
\hline
\end{tabular}


Tabela 6. Equilíbrio químico dos solutos e equações de balanço de massa e carga para as espécies de manganês. Adaptada das refs. 23-28

\begin{tabular}{|c|c|}
\hline Reação & Constante de equilíbrio \\
\hline $\mathrm{H}_{2} \mathrm{O}=\mathrm{H}^{+}+\mathrm{OH}^{-}$ & $\mathrm{K}_{1}=1,00 \times 10^{-14}$ \\
\hline $\mathrm{H}^{+}+\mathrm{SO}_{4}^{2-}=\mathrm{HSO}_{4}^{-}$ & $\mathrm{K}_{2}=9,85 \times 10^{1}$ \\
\hline $\begin{aligned} \mathrm{Mn}^{2+}+\mathrm{SO}_{4}{ }^{2-} & =\mathrm{MnSO}_{4(\mathrm{aq})} \\
\mathrm{Ni}^{2+}+\mathrm{SO}_{4}{ }^{2-} & =\mathrm{NiSO}_{4(\mathrm{aq})} \\
\mathrm{Co}^{2+}+\mathrm{SO}_{4}{ }^{2-} & =\mathrm{CoSO}_{4(\mathrm{aq})} \\
\mathrm{Cu}^{2+}+\mathrm{SO}_{4}{ }^{2-} & =\mathrm{CuSO}_{4(\mathrm{aq})}\end{aligned}$ & $\begin{array}{c}\mathrm{K}_{3}=190 \\
\mathrm{~K}_{4}=1,92 \times 10^{2} \\
\mathrm{~K}_{5}=1,97 \times 10^{2} \\
\mathrm{~K}_{6}=251,2\end{array}$ \\
\hline $\begin{aligned} \mathrm{Mn}^{2+}+\mathrm{HSO}_{4} & =\mathrm{MnHSO}_{4}{ }^{+} \\
\mathrm{Ni}^{2+}+\mathrm{HSO}_{4} & =\mathrm{NiHSO}_{4}^{+} \\
\mathrm{Co}^{2+}+\mathrm{HSO}_{4}^{-} & =\mathrm{CoHSO}_{4}^{+} \\
\mathrm{Cu}^{+2}+\mathrm{HSO}_{4} & =\mathrm{CuHSO}_{4}^{+}\end{aligned}$ & $\begin{array}{c}\mathrm{K}_{7}=181,97 \\
\mathrm{~K}_{8}=2,18 \times 10^{2} \\
\mathrm{~K}_{9}=2,19 \times 10^{2} \\
\mathrm{~K}_{10}=229,1\end{array}$ \\
\hline $\begin{aligned} \mathrm{Mn}^{2+}+\mathrm{OH}^{-} & =\mathrm{MnOH}^{+} \\
\mathrm{Ni}^{2+}+\mathrm{OH}^{-} & =\mathrm{NiOH}^{+} \\
\mathrm{Co}^{2+}+\mathrm{OH}^{-} & =\mathrm{CoOH}^{+} \\
\mathrm{Cu}^{2+}+\mathrm{OH}^{-} & =\mathrm{CuOH}^{+}\end{aligned}$ & $\begin{aligned} \mathrm{K}_{11} & =6,27 \times 10^{3} \\
\mathrm{~K}_{12} & =1,26 \times 10^{4} \\
\mathrm{~K}_{13} & =1,99 \times 10^{4} \\
\mathrm{~K}_{14} & =5,01 \times 10^{7}\end{aligned}$ \\
\hline $\begin{aligned} \mathrm{Mn}^{2+}+2 \mathrm{OH}^{-} & =\mathrm{Mn}(\mathrm{OH})_{2} \\
\mathrm{Ni}^{2+}+2 \mathrm{OH}^{-} & =\mathrm{Ni}(\mathrm{OH})_{2} \\
\mathrm{Co}^{2+}+2 \mathrm{OH}^{-} & =\mathrm{Co}(\mathrm{OH})_{2} \\
\mathrm{Cu}^{2+}+2 \mathrm{OH}^{-} & =\mathrm{Cu}(\mathrm{OH})_{2}\end{aligned}$ & $\begin{array}{l}\mathrm{K}_{15}=6,31 \times 10^{12} \\
\mathrm{~K}_{16}=1,00 \times 10^{8} \\
\mathrm{~K}_{17}=2,51 \times 10^{8} \\
\mathrm{~K}_{18}=1,58 \times 10^{16}\end{array}$ \\
\hline
\end{tabular}

Equações de balanço de massa

$[\mathrm{Mn}]^{\mathrm{i}}=\left[\mathrm{Mn}^{2+}\right]+\left[\mathrm{MnSO}_{4}\right]+\left[\mathrm{MnHSO}_{4}^{+}\right]+\left[\mathrm{MnOH}^{+}\right]+\left[\mathrm{Mn}(\mathrm{OH})_{2}\right]+\left(\mathrm{V}_{\mathrm{or}} / \mathrm{V}_{\mathrm{ac}}\right)\left[\mathrm{MnH}_{2 \mathrm{x}} \mathrm{A}_{2+2 \mathrm{x}}\right]$ $[\mathrm{Nii}]^{\mathrm{i}}=\left[\mathrm{Ni}^{2+}\right]+\left[\mathrm{NiSO}_{4}\right]+\left[\mathrm{NiHSO}_{4}^{+}\right]+\left[\mathrm{NiOH}^{+}\right]+\left[\mathrm{Ni}(\mathrm{OH})_{2}\right]+\left(\mathrm{V}_{\mathrm{or}} / \mathrm{V}_{\mathrm{aq}}\right)\left[\mathrm{NiH}_{2 \mathrm{X}} \mathrm{A}_{2+2 \mathrm{x}}\right]$ $[\mathrm{Co}]^{\mathrm{i}}=\left[\mathrm{Co}^{2+}\right]+\left[\mathrm{CoSO}_{4}\right]+\left[\mathrm{CoHSO}_{4}^{+}\right]+\left[\mathrm{CoOH}^{+}\right]+\left[\mathrm{Co}(\mathrm{OH})_{2}\right]+\left(\mathrm{V}_{\mathrm{og}} / \mathrm{V}_{\mathrm{aq}}\right)\left[\mathrm{CoH}_{2 \times} \mathrm{A}_{2+2 \mathrm{x}}\right]$ $[\mathrm{Cu}]^{i}=\left[\mathrm{Cu}^{2+}\right]+\left[\mathrm{CuSO}_{4}\right]+\left[\mathrm{CuHSO}_{4}^{+}\right]+\left[\mathrm{CuOH}^{+}\right]+\left[\mathrm{Cu}(\mathrm{OH})_{2}\right]+\left(\mathrm{V}_{\mathrm{or}} / \mathrm{V}_{\mathrm{aq}}\right)\left[\mathrm{CuH}_{2 \mathrm{x}} \mathrm{A}_{2+2 \mathrm{x}}\right]$

Equações de balanço de carga

$\left[\mathrm{H}^{+}\right]+\left[\mathrm{MnHSO}_{4}^{+}\right]+\left[\mathrm{MnOH}^{+}\right]+2\left[\mathrm{Mn}^{2+}\right]=\left[\mathrm{OH}^{-}\right]+\left[\mathrm{HSO}_{4}{ }^{-}\right]+2\left[\mathrm{SO}_{4}{ }^{2-}\right]$

$\left[\mathrm{H}^{+}\right]+\left[\mathrm{NiHSO}_{4}^{+}\right]+\left[\mathrm{NiOH}^{+}\right]+2\left[\mathrm{Ni}^{2+}\right]=\left[\mathrm{OH}^{-}\right]+\left[\mathrm{HSO}_{4}{ }^{-}\right]+2\left[\mathrm{SO}_{4}{ }^{2-}\right]$

$\left[\mathrm{H}^{+}\right]+\left[\mathrm{CoHSO}_{4}^{+}\right]+\left[\mathrm{CoOH}^{+}\right]+2\left[\mathrm{Co}^{2+}\right]=\left[\mathrm{OH}^{-}\right]+\left[\mathrm{HSO}_{4}{ }^{-}\right]+2\left[\mathrm{SO}_{4}{ }^{2-}\right]$

$\left[\mathrm{H}^{+}\right]+\left[\mathrm{CuHSO}_{4}^{+}\right]+\left[\mathrm{CuOH}^{+}\right]+2\left[\mathrm{Cu}^{2+}\right]=[\mathrm{OH}]+\left[\mathrm{HSO}_{4}^{-}\right]+2\left[\mathrm{SO}_{4}^{2-}\right]$

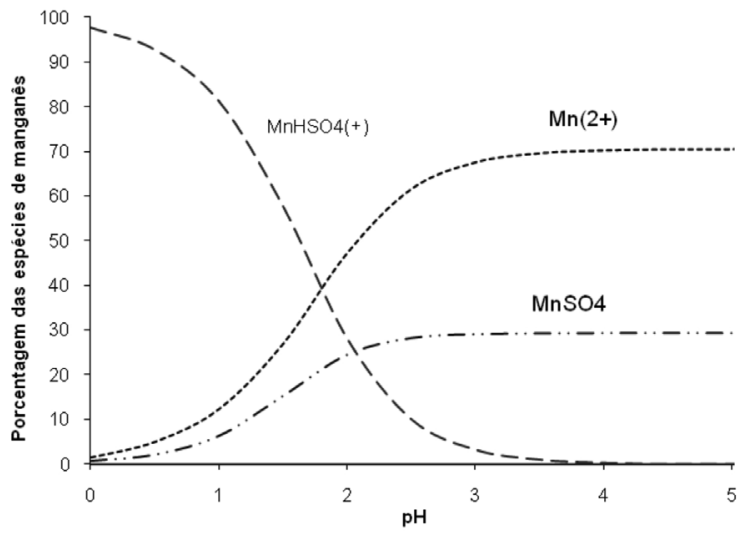

Figura 1. Gráfico da especiação do manganês construído pelo modelo termodinâmico

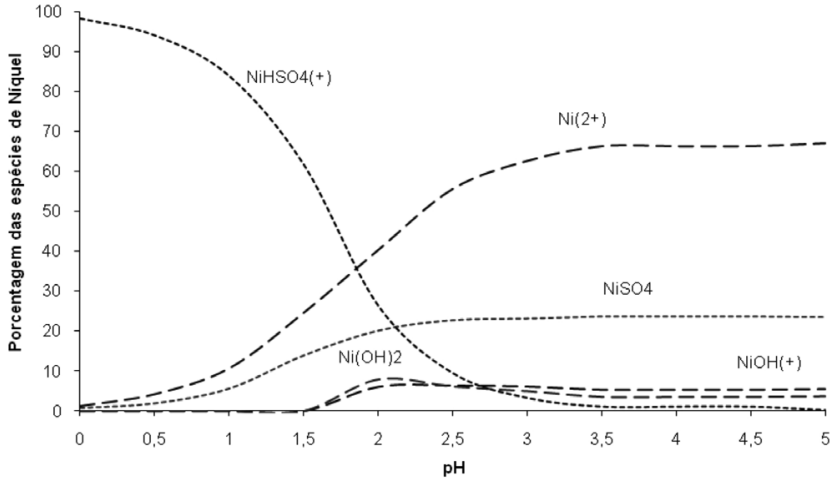

Figura 2. Gráfico da especiação do níquel construído pelo modelo termodinâmico

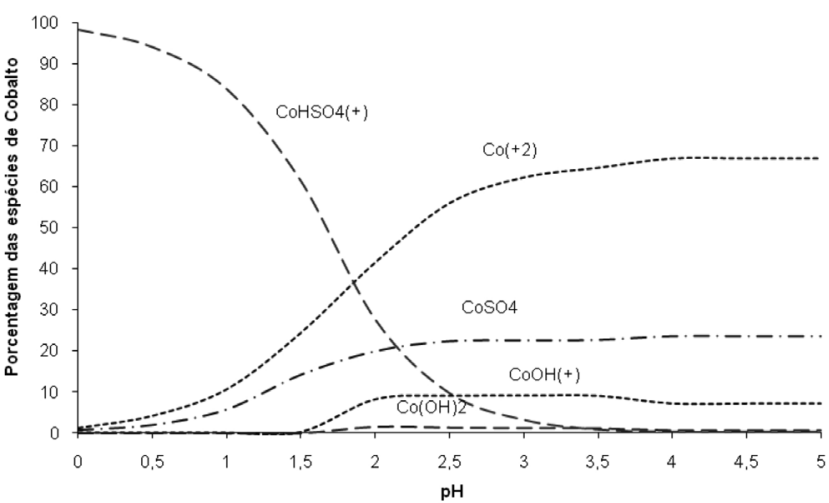

Figura 3. Gráfico da especiação do cobalto construído pelo modelo termodinâmico

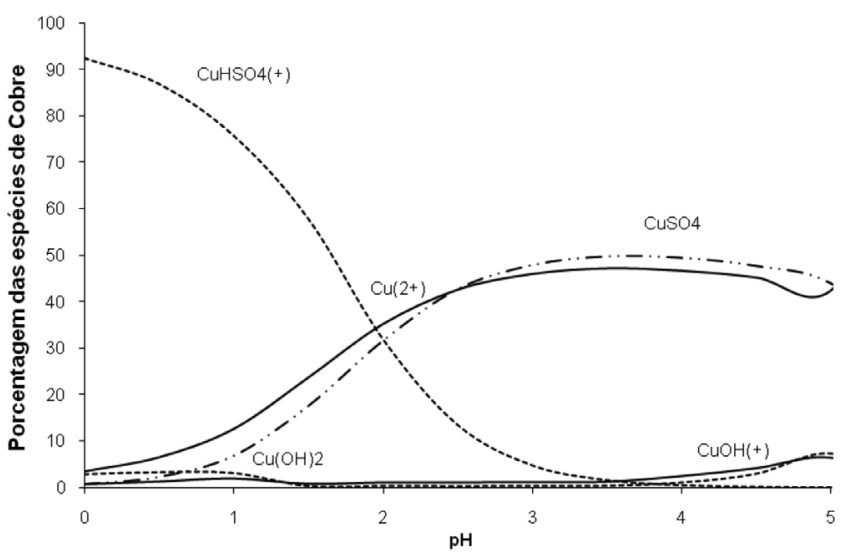

Figura 4. Gráfico da especiação do cobre construído pelo modelo termodinâmico

Tabela 7. Força iônica e coeficientes de atividades para cada íon aquoso de manganês em solução

\begin{tabular}{cccccccccc}
\hline $\mathrm{pH}$ & Força iônica & $\mathrm{Mn}^{2+}$ & $\mathrm{MnSO}_{4}$ & $\mathrm{MnHSO}_{4}^{+}$ & $\mathrm{MnOH}^{+}$ & $\mathrm{Mn}(\mathrm{OH})_{2}$ & $\mathrm{OH}^{-}$ & $\mathrm{SO}_{4}^{2-}$ & $\mathrm{HSO}_{4}^{-}$ \\
\hline 0 & 1261,3572 & 0,2745 & 1,0000 & 0,7238 & 0,7238 & 1,0000 & 0,7238 & 0,2745 \\
0,5 & 466,1663 & 0,2360 & 1,0000 & 0,6970 & 0,6970 & 1,0000 & 0,6970 & 0,2360 & 0,7238 \\
1 & 178,4511 & 0,3006 & 1,0000 & 0,7404 & 0,7404 & 1,0000 & 0,7404 & 0,3006 & 0,7404 \\
1,5 & 79,1534 & 0,3926 & 1,0000 & 0,7915 & 0,7915 & 1,0000 & 0,7915 & 0,3926 & 0,7915 \\
2 & 46,8782 & 0,4616 & 1,0000 & 0,8243 & 0,8243 & 1,0000 & 0,8243 & 0,4616 & 0,8243 \\
2,5 & 38,1279 & 0,4898 & 1,0000 & 0,8366 & 0,8366 & 1,0000 & 0,8366 & 0,4898 & 0,8366 \\
3 & 35,6052 & 0,4992 & 1,0000 & 0,8406 & 0,8406 & 1,0000 & 0,8406 & 0,4992 \\
3,5 & 35,0325 & 0,5014 & 1,0000 & 0,8415 & 0,8415 & 1,0000 & 0,8415 & 0,8406 \\
4 & 34,7886 & 0,5024 & 1,0000 & 0,8419 & 0,8419 & 1,0000 & 0,8419 & 0,5024 & 0,8415 \\
4,5 & 34,8239 & 0,5022 & 1,0000 & 0,8418 & 0,8418 & 1,0000 & 0,8418 & 0,8419 \\
5 & 34,7969 & 0,5024 & 1,0000 & 0,8419 & 0,8419 & 1,0000 & 0,8419 & 0,5024 & 0,8418 \\
\hline
\end{tabular}


Tabela 8. Força iônica e coeficientes de atividades para cada íon aquoso de níquel em solução

\begin{tabular}{|c|c|c|c|c|c|c|c|c|c|}
\hline $\mathrm{pH}$ & Força iônica & $\mathrm{Ni}^{2+}$ & $\mathrm{NiSO}_{4}$ & $\mathrm{NiHSO}_{4}^{+}$ & $\mathrm{NiOH}^{+}$ & $\mathrm{Ni}(\mathrm{OH})_{2}$ & $\mathrm{OH}^{-}$ & $\mathrm{SO}_{4}^{2-}$ & $\mathrm{HSO}_{4}^{-}$ \\
\hline 0 & $1,26 \times 10^{3}$ & 0,274 & 1,000 & 0,724 & 0,724 & 1,000 & 0,724 & 0,274 & 0,724 \\
\hline 0,5 & $4,62 \times 10^{2}$ & 0,236 & 1,000 & 0,697 & 0,697 & 1,000 & 0,697 & 0,236 & 0,697 \\
\hline 1,0 & $1,73 \times 10^{2}$ & 0,304 & 1,000 & 0,742 & 0,742 & 1,000 & 0,742 & 0,304 & 0,742 \\
\hline 1,5 & $7,24 \times 10^{1}$ & 0,404 & 1,000 & 0,797 & 0,797 & 1,000 & 0,797 & 0,404 & 0,797 \\
\hline 2,0 & $3,61 \times 10^{1}$ & 0,497 & 1,000 & 0,840 & 0,840 & 1,000 & 0,840 & 0,497 & 0,840 \\
\hline 2,5 & $2,65 \times 10^{1}$ & 0,540 & 1,000 & 0,857 & 0,857 & 1,000 & 0,857 & 0,540 & 0,857 \\
\hline 3,0 & $2,41 \times 10^{1}$ & 0,552 & 1,000 & 0,862 & 0,862 & 1,000 & 0,862 & 0,552 & 0,862 \\
\hline 3,5 & $2,39 \times 10^{1}$ & 0,554 & 1,000 & 0,863 & 0,863 & 1,000 & 0,863 & 0,554 & 0,863 \\
\hline 4,0 & $2,38 \times 10^{1}$ & 0,560 & 1,000 & 0,865 & 0,865 & 1,000 & 0,865 & 0,560 & 0,865 \\
\hline 4,5 & $2,35 \times 10^{1}$ & 0,562 & 1,000 & 0,865 & 0,865 & 1,000 & 0,865 & 0,562 & 0,865 \\
\hline 5,0 & $2,35 \times 10^{1}$ & 0,563 & 1,000 & 0,869 & 0,869 & 1,000 & 0,869 & 0,563 & 0,869 \\
\hline
\end{tabular}

Tabela 9. Força iônica e coeficientes de atividades para cada íon aquoso de cobalto em solução

\begin{tabular}{|c|c|c|c|c|c|c|c|c|c|}
\hline $\mathrm{pH}$ & Força iônica & $\mathrm{Co}^{2+}$ & $\mathrm{CoSO}_{4}$ & $\mathrm{CoHSO}_{4}^{+}$ & $\mathrm{CoOH}^{+}$ & $\mathrm{Co}(\mathrm{OH})_{2}$ & $\mathrm{OH}^{-}$ & $\mathrm{SO}_{4}^{2-}$ & $\mathrm{HSO}_{4}$ \\
\hline 0 & $1,26 \times 10^{3}$ & 0,274 & 1,000 & 0,724 & 0,724 & 1,000 & 0,724 & 0,274 & 0,724 \\
\hline 0,5 & $4,62 \times 10^{2}$ & 0,236 & 1,000 & 0,697 & 0,697 & 1,000 & 0,697 & 0,236 & 0,697 \\
\hline 1,0 & $1,73 \times 10^{2}$ & 0,304 & 1,000 & 0,742 & 0,742 & 1,000 & 0,742 & 0,304 & 0,742 \\
\hline 1,5 & $7,23 \times 10^{1}$ & 0,404 & 1,000 & 0,797 & 0,797 & 1,000 & 0,797 & 0,404 & 0,797 \\
\hline 2,0 & $3,74 \times 10^{1}$ & 0,492 & 1,000 & 0,838 & 0,838 & 1,000 & 0,838 & 0,492 & 0,838 \\
\hline 2,5 & $2,74 \times 10^{1}$ & 0,535 & 1,000 & 0,855 & 0,855 & 1,000 & 0,855 & 0,535 & 0,855 \\
\hline 3,0 & $2,46 \times 10^{1}$ & 0,550 & 1,000 & 0,861 & 0,861 & 1,000 & 0,861 & 0,550 & 0,861 \\
\hline 3,5 & $2,39 \times 10^{1}$ & 0,554 & 1,000 & 0,863 & 0,863 & 1,000 & 0,863 & 0,554 & 0,863 \\
\hline 4,0 & $2,41 \times 10^{1}$ & 0,553 & 1,000 & 0,862 & 0,862 & 1,000 & 0,862 & 0,553 & 0,862 \\
\hline 4,5 & $2,34 \times 10^{1}$ & 0,556 & 1,000 & 0,864 & 0,864 & 1,000 & 0,864 & 0,556 & 0,864 \\
\hline 5,0 & $2,36 \times 10^{1}$ & 0,555 & 1,000 & 0,863 & 0,863 & 1,000 & 0,863 & 0,555 & 0,863 \\
\hline
\end{tabular}

Tabela 10. Força iônica e coeficientes de atividades para cada íon aquoso de cobre em solução

\begin{tabular}{|c|c|c|c|c|c|c|c|c|c|}
\hline $\mathrm{pH}$ & Força iônica & $\mathrm{Cu}^{2+}$ & $\mathrm{CuSO}_{4}$ & $\mathrm{CuHSO}_{4}^{+}$ & $\mathrm{CuOH}^{+}$ & $\mathrm{Cu}(\mathrm{OH})_{2}$ & $\mathrm{OH}^{-}$ & $\mathrm{SO}_{4}^{2-}$ & $\mathrm{HSO}_{4}$ \\
\hline 0 & $5,07 \times 10^{3}$ & 0,234 & 1,000 & 0,695 & 0,695 & 1,000 & 0,695 & 0,234 & 0,695 \\
\hline 0,5 & $1,64 \times 10^{3}$ & 0,309 & 1,000 & 0,745 & 0,745 & 1,000 & 0,745 & 0,309 & 0,745 \\
\hline 1,0 & $5,66 \times 10^{2}$ & 0,436 & 1,000 & 0,813 & 0,813 & 1,000 & 0,813 & 0,436 & 0,813 \\
\hline 1,5 & $2,34 \times 10^{2}$ & 0,556 & 1,000 & 0,864 & 0,864 & 1,000 & 0,864 & 0,556 & 0,864 \\
\hline 2,0 & $1,34 \times 10^{2}$ & 0,630 & 1,000 & 0,891 & 0,891 & 1,000 & 0,891 & 0,630 & 0,891 \\
\hline 2,5 & $1,04 \times 10^{2}$ & 0,661 & 1,000 & 0,902 & 0,902 & 1,000 & 0,902 & 0,661 & 0,902 \\
\hline 3,0 & $9,55 \times 10^{1}$ & 0,671 & 1,000 & 0,905 & 0,905 & 1,000 & 0,905 & 0,671 & 0,905 \\
\hline 3,5 & $9,27 \times 10^{1}$ & 0,675 & 1,000 & 0,906 & 0,906 & 1,000 & 0,906 & 0,675 & 0,906 \\
\hline 4,0 & $9,04 \times 10^{1}$ & 0,678 & 1,000 & 0,907 & 0,907 & 1,000 & 0,907 & 0,678 & 0,907 \\
\hline 4,5 & $8,74 \times 10^{1}$ & 0,681 & 1,000 & 0,909 & 0,909 & 1,000 & 0,909 & 0,682 & 0,909 \\
\hline 5,0 & $8,21 \times 10^{1}$ & 0,690 & 1,000 & 0,911 & 0,911 & 1,000 & 0,911 & 0,689 & 0,911 \\
\hline
\end{tabular}

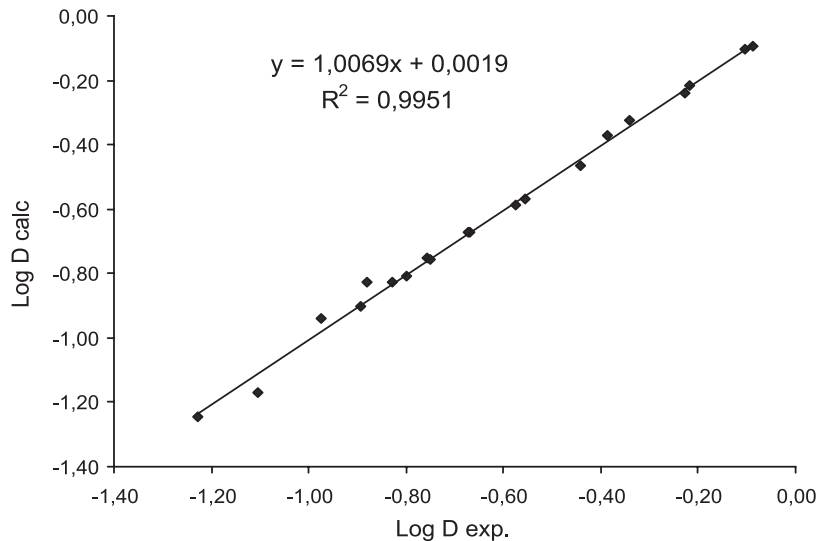

Figura 5. Valores de $\log D$ calculado versus $\log D$ experimental para as soluções de manganês

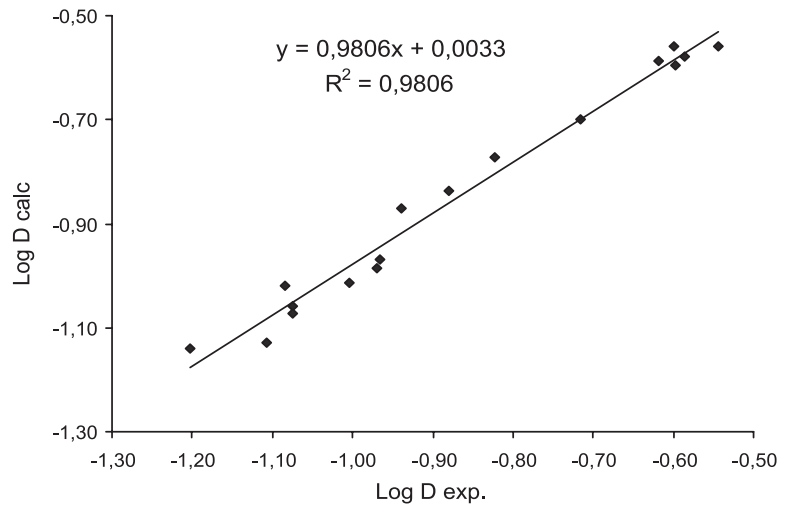

Figura 6. Valores de $\log D$ calculado versus $\log D$ experimental para as soluções de níquel 


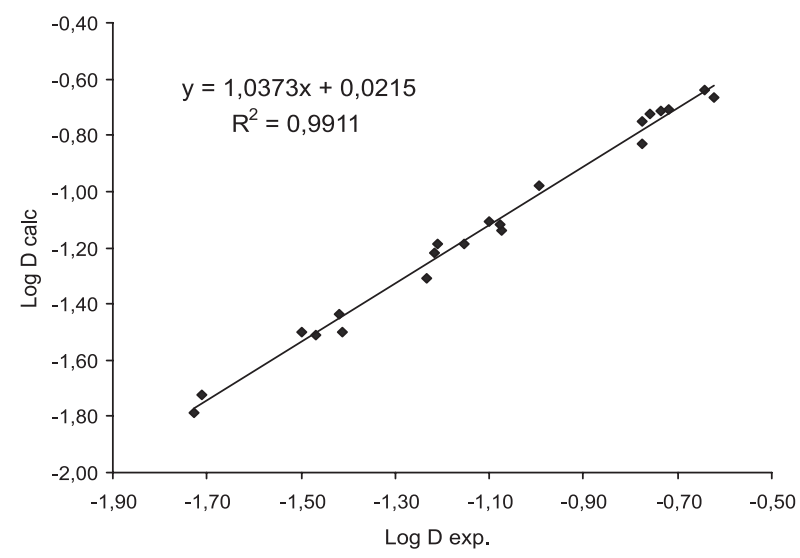

Figura 7. Valores de $\log D$ calculado versus $\log D$ experimental para as soluções de cobalto

Após extração com D2EHPA as espécies químicas são treze $\left(\mathrm{M}^{2+}\right.$, $\mathrm{MSO}_{4}, \mathrm{MHSO}_{4}{ }^{+}, \mathrm{MOH}^{+}, \mathrm{M}(\mathrm{OH})_{2}, \mathrm{H}^{+}, \mathrm{OH}^{-}, \mathrm{SO}_{4}{ }^{2-}, \mathrm{HSO}_{4}{ }^{+}, \mathrm{H}_{2} \mathrm{O}, \mathrm{H}_{2} \mathrm{~A}_{2}$ $\mathrm{MA}_{2}$ e diluente). Os valores de coeficiente de atividade para todas as espécies incluindo $\mathrm{H}_{2} \mathrm{O}$ na fase orgânica foram considerados iguais a 1 , devido à ausência de dados na literatura. Então, 11 equações independentes são necessárias para calcular a atividade das onze espécies químicas e esta será obtida a partir de sete equilíbrios químicos e um balanço de massa e um balanço da carga. O método de NewtonRaphson é novamente utilizado e a metodologia do cálculo é a mesma descrita acima. Para verificar a validade do modelo, os valores de log D obtidos experimentalmente e aqueles calculados pelo modelo estão dispostos graficamente nas Figuras 5 a 8 . A análise estatística mostra um bom ajuste dos modelos termodinâmicos quando são comparados com os dados experimentais, com um nível de confiança de $95 \%$ e um coeficiente de correlação de 0,9951; 0,9806; 0,9911 e 0,9855, respectivamente, para manganês, níquel, cobalto e cobre.

\section{CONCLUSÕES}

Os estudos mostraram que as espécies aquosas extraídas de manganês, níquel, cobalto e cobre foram $\mathrm{MnH}_{0,2} \mathrm{~A}_{2,2}, \mathrm{NiH}_{0,25} \mathrm{~A}_{2,25}$, $\mathrm{CoH}_{0,25} \mathrm{~A}_{2,25}$ e $\mathrm{CuH}_{2,4} \mathrm{~A}_{4,4}$ (onde HA é D2EHPA) e para a constante de equilíbrio calculada com a extração de dados experimentais, utilizando Quasi-Newton regressão método não linear, foram encontrados valores de $5,18 \times 10^{-4} ; 2,40 \times 10^{-5} ; 2,10 \times 10^{-5}$ e $2,52 \times 10^{-1}$, respectivamente. Os valores de $\mathrm{K}_{\mathrm{eq}}$ confirmam que, para metais de mesma carga, a extração é inversamente proporcional ao raio iônio, pois quanto menor o íon, mais efetiva será a extração.

As concentrações e os coeficientes de atividade das espécies em solução antes da extração foram calculados utilizando-se o valor do $\mathrm{pH}$ e a concentração total de manganês, níquel, cobalto e cobre foi calculada usando-se a Equação de Davies modificada. Os valores dos coeficientes de distribuição previstos pelo modelo para a extração de $\mathrm{Mn}(\mathrm{II}), \mathrm{Ni}(\mathrm{II}), \mathrm{Co}(\mathrm{II})$ e $\mathrm{Cu}(\mathrm{II})$ estão em boa concordância quando comparados com resultados experimentais.

\section{REFERÊNCIAS}

1. Owusu, G.; Hydrometallurgy 1998, 47, 205.

2. Tsakiridis, P. E.; Agatzini, S. L.; Hydrometallurgy 2004, 72, 269.

3. Tanaka, M.; Hydrometallurgy 1990, 24, 317.

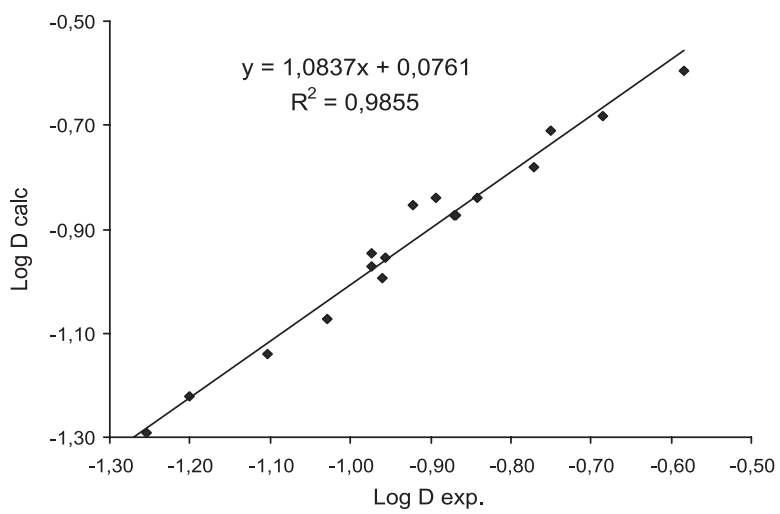

Figura 8. Valores de log D calculado versus $\log D$ experimental para as soluções de cobre

4. Valverde Jr., I. M.; Paulino, J. F.; Afonso, J. C.; Quim. Nova 2008, 31, 680.

5. Lee, M.; Ahn, J.; Son, S.; Materials Transactions 2001, 42, 2548.

6. Samson, E.; Lemaire, G.; Marchand, J.; Beaudoin, J.J.; Computational Materials Science 1999, 15, 285.

7. Pitzer, K. S.; Mayorga, G.; J. Phys. Chemical 1973, 77, 2300.

8. Bromley, L. A.; AIChE J. 1973, 19, 313.

9. Beutier, D.; Renon, H.; IEC. Proc. Des. Dev. 1978, 17, 220.

10. Juang, R. S,; Su, J. Y.; IEC. Res. 1992, 31, 2395.

11. Biembengut, M. S.; Hein, N.; Modelagem Matemática no Ensino, $3^{\text {a }}$ ed., Contexto: São Paulo, 2003.

12. Butler, J. N.; Cogley, D. R.; Ionic Equilibrium - Solubility and pH calculations, John Wiley \& Sons: New York, 1998.

13. Forrest, C.; Hughes, M. A.; Hydrometallurgy 1975, 1, 25.

14. Swain, B.; Jeong, J.; Lee, J.; Lee, G. H.; Hydrometallurgy 2006, 84, 130.

15. Fu, X.; Golding, J. A.; Solvent Extr. Ion Exch. 1987, 5, 205.

16. Biswas, R. K.; Begum, D. A.; Hydrometallurgy 2000, 55, 57.

17. Monhenius, A. J.; An introduction to metallurgical solvent extraction, COPPE/ UFRJ: Rio de Janeiro, 1975.

18. Ritcey, M. G.; Ashbrook, A. W.; Solvent Extraction: principles and applications to process metallurgy, $1^{\text {st }}$ ed., Elsevier Scientific Publishing Company: Netherlands, 1979.

19. Pitzer, K. S.; Phys. Chem. Earth 1973, 13, 249.

20. Sousa Junior, C. S.; Nascimento, M.; Masson, I. O. C.; Cunha, O. G. C.; Hydrometallurgy 2010, 103, 114.

21. Atkins, P.; De Paula, J.; Físico-Química, $8^{\mathrm{a}}$ ed., Editora LTC: São Paulo, 2008.

22. Ritcey, G. M.; Ashbrook, A. W.; Solvent Extraction: principles and aplications to process metallurgy, $2^{\text {nd }}$ ed., Elsevier Scientific Publishing Company: Netherlands, 1984.

23. Smith, R. M.; Martell, A. E.; Critical stability constants, Plenum: New York, 1982.

24. Bjerrum, J.; Schwarzenbach, G.; Sillen, L. G.; Stability Constants of Metal-ion Complexes, $7^{\text {th }}$ ed., The Chemical Society: London, 1957.

25. Morel, F,; Hering, J. G.; Principles and applications of aquatic chemistry, John Wiley and Sons: New York, 1993.

26. Senanayake, G.; Hydrometallurgy 2004, 75, 55.

27. Plyasunova, N. V.; Zhang, Y.; Muhammed, M.; Hydrometallurgy 1998, $48,43$.

28. Scientific Group Thermodata Europe; In Thermodynamic Properties of Inorganic Material; Landolt-Börnstein, New Series, Gourp IV, vol. 19, edit by Lehrsyuhl Für Theoretische: Berlin, 1999. 